\title{
Initialization of the shallow water equations with open boundaries by the bounded derivative method
}

\author{
By G. BROWNING, National Center for Atmospheric Research, ${ }^{1}$ P.O. Box 3000, Boulder, Colorado \\ 80307, U.S.A. and H.-O. KREISS, ${ }^{2}$ Applied Mathematics, California Institute of Technology, Pasadena, \\ California 91125, U.S.A.
}

(Manuscript received June 10: in final form December 14, 1981)

\begin{abstract}
The shallow water equations are a symmetric hyperbolic system with two time scales. In meteorological terms, slow and fast scale motions are referred to as Rossby and inertial/gravity waves, respectively. We prove the existence of smooth solutions (solutions with a number of space and time derivatives on the order of the slow time scale) of the open boundary problem for the shallow water equations by the bounded derivative method. The proof requires that a number of initial time derivatives be of the order of the slow time scale and that the boundary data be smooth. If the boundary data are smooth and only have small errors, then we show that the solution of the open boundary problem is smooth and that only small errors are produced in the interior. If the boundary data are smooth but have large errors, then we show that the solution of the open boundary problem is still smooth. Unfortunately the boundary error propagates into the interior at the speed associated with the fast time scale and destroys the solution in a short time. Thus it is necessary to keep the boundary error small if the solution is to be computed correctly. We show that this restriction can be relaxed so that only the large-scale boundary data need be correct. We demonstrate the importance of these conclusions in several numerical experiments.
\end{abstract}

\section{Introduction}

In a previous paper (Browning et al., 1980) we proved the existence of smooth solutions of the equations of shallow water flow in a channel by the bounded derivative method (Kreiss, 1980). We then showed how to determine the initial data to obtain such a solution and demonstrated the effectiveness of the technique in a numerical model. However, in that paper we did not treat the case of open boundaries. The case of open boundaries is of considerable interest for a number of reasons. First. the high resolution which is needed to handle detailed synoptic features, such as atmospheric fronts, cannot be used in a global model with the current level of computing power. In fact a global high resolution grid should not be used, even if

\footnotetext{
' Sponsored by the National Science Foundation.

${ }^{2}$ Supported by the Office of Naval Research under Contract No. N00014-80-C0076.
}

practical, as fronts are a local phenomenon and should be handled adaptively (Berger et al., 1981). Also, observations of synoptic data are sufficiently dense to define the scales appropriate for a high resolution model only in certain areas of the globe. For all of these reasons, a considerable amount of research investigating different candidates for open boundary conditions has appeared in the literature. An excellent survey of this subject is given in Sundström and Elvius (1979).

The theory of boundary conditions for the open boundary problem for symmetric hyperbolic systems is now well developed (Kreiss, 1970). If a set of well-posed boundary conditions for such a system is of the right form, then the bounded derivative method can be used to prove the existence of smooth solutions and an initialization procedure to obtain these solutions can be derived. As an introduction to the concepts and techniques of the bounded derivative method for open boundary problems, in Section 2 we apply the bounded 
derivative method to an initial-boundary value problem for a simple symmetric hyperbolic system. Then following the techniques used in Section 2, we prove the existence of smooth solutions of the shallow water equations for a particular choice of well-posed open boundary conditions and derive the corresponding initialization procedure in Sections 3 and 4 , respectively. In Section 5 we demonstrate the effectiveness of the initialization procedure in a numerical model and some of the pitfalls of limited area modeling. Conclusions are presented in Section 6.

\section{Open boundaries and the bounded derivative method}

As an introduction to the application of the bounded derivative method to problems with open boundaries (Kreiss, 1980), we consider the symmetric hyperbolic system

$Z_{t}+\left[\begin{array}{cc}\bar{u} & 0 \\ 0 & \varepsilon^{-1} \bar{v}\end{array}\right] Z_{x}=\left[\begin{array}{l}0 \\ f\end{array}\right]$,

where $\bar{u}$ and $\bar{v}$ are nonzero constants of order unity, $\varepsilon$ is a constant such that $0<\varepsilon \ll 1, f(x, t)$ is a known smooth function (smoothness means that the function and a number of its space and time derivatives have norms of order unity), and

$Z(x, t)=\left[\begin{array}{l}u \\ v\end{array}\right]$.

This is the simplest hyperbolic system which contains multiple time scales and which suffices to demonstrate how open boundaries are taken into account when applying the bounded derivative method. We now seek estimates of the solutions of (2.1) which show that smooth solutions of (2.1) exist, i.e. the bound on the norm of the solution must only involve constants of order unity. If we can show that the constants in the bound on the solution operator of the homogeneous version of (2.1) are of order unity, then we can also obtain estimates for the solutions of (2.1) of the desired form by Duhamel's principle (John, 1952). Then in the estimation process we can assume $f=0$ without loss of generality.

We first consider system (2.1) on the onedimensional torus, i.e. we assume that $0 \leqslant x \leqslant 2 \pi$ with $f$ and $Z$ being $2 \pi$-periodic in $x$. Multiplying the homogeneous version of (2.1) by $Z^{T}$ and then integrating the resulting equation with respect to $x$ over the entire circle, we obtain the equality $\|Z(x, t)\|=$ $\|Z(x, 0)\|\left(\|\cdot\|\right.$ denotes the usual $L^{2}$ norm) so that the norm of the solution operator $S$ satisfies $\|S\|=1$. From this equality and the fact that any time derivative of $Z$ satisfies the same equation as $Z$ itself, we can conclude that if the norm of any of the initial time derivatives of $Z$ is of order unity, then it will remain so for all time. Since $\bar{u}$ and $\bar{v}$ are assumed to be nonzero, we can solve (2.1) for $Z_{x}$ in terms of $Z_{t}$ so that if the norm of the first-order time derivative is of order unity, then the norm of the first-order space derivative is also of order unity. A similar argument holds for the higher order space derivatives of $Z$ so that we only need to consider the initial time derivatives of $Z$.

For the toroidal problem the two equations of (2.1) uncouple. The first equation is the one whose solution propagates with a speed of order unity. If the initial data for this equation are smooth, then all the initial time derivatives of $u$ will be of order unity. The homogeneous version of the second equation has solutions which propagate with a speed of order $\varepsilon^{-1}$. We classify the solutions of this equation into two types. The first type of solution of the form $v(x, t)=v_{0}\left(x-\varepsilon^{-1} \bar{v} t\right)$ has time derivatives which are not of order unity even if the initial value $v_{0}(x)$ of $v$ is smooth. This type of solution is always to be suppressed. The second type of solution of the form $v(x, t)=v_{0}(\varepsilon x-\bar{v} t)$ is smooth if the initial value $v_{0}(\varepsilon x)$ of $v$ is smooth. In practical applications this type of solution is an important component of the desired solution and must not be eliminated. This fact has serious implications for initial-boundary value problems as we shall see later on. We now show how to ensure that a number of initial time derivatives of $v$ are of order unity so that the ensuing solution will be smooth as shown by the proof above.

The first-order time derivative of $v$ is of order unity if and only if

$v_{x}(x, t)=\varepsilon a(x, t)$,

where $a$ is a given function whose norm is of order unity. To ensure a periodic solution of (2.2) exists, we assume that the mean of $a$ is zero, i.e.

$(2 \pi)^{-1} \int_{0}^{2 \pi} a(x, t) d x=0$.

Then (2.2) uniquely determines $v$ except for its mean value. In the limit as $\varepsilon \rightarrow 0,(2.2)$ requires that 
$v$ be independent of $x$ and it is exactly the mean value of $v$ that survives. This can also be seen by taking the limit as $\varepsilon \rightarrow 0$ of the second type of solution of the homogeneous equation (2.1) for $v$. By integrating the second equation of $(2,1)$ with respect to $x$ from 0 to $2 \pi$, we see that the mean of $v$ is a solution of the equation

$\frac{\partial}{\partial t} \int_{0}^{2 \pi} v \mathrm{~d} x=\int_{0}^{2 \pi} f \mathrm{~d} x$

and, therefore, is completely determined by the mean of $f$ and the mean of the initial data for $v$. With this value for the mean of $v,(2.2)$ has a unique solution.

Using (2.2) we rewrite the second equation of (2.1) in the form

$v_{\iota}+\bar{v} a=f$,

so that the second-order time derivative of $v$ is given by

$v_{t t}+\bar{v} a_{t}=f_{t}$

Since we have assumed that $f$ is smooth, the second-order time derivative of $v$ is of order unity if and only if $a_{t}$ is of order unity. But by (2.2) and (2.3)

$-\varepsilon a_{t}=-v_{x t}=(\bar{v} a-f)_{x}$.

Then the second-order time derivative of $v$ is of order unity if and only if

$(\bar{v} a-f)_{x}=\varepsilon b(x, t)$,

where $b$ is a given function whose norm is of order unity and whose mean is zero. This constraint uniquely determines $a$ to within an error term of order $\varepsilon$. We can use the solution of (2.4) in the previous constraint (2.2) to determine $v$ uniquely to within an error term of order $\varepsilon^{2}$. Clearly this procedure can be carried out indefinitely.

The above procedure enables us to choose the initial data for system (2.1) so that the ensuing solution is smooth. There is another approach that can also be taken. As we will need to use some of the results from that approach, we will also briefly discuss that theory. In that approach the nonsmooth solutions are eliminated entirely by deriving the corresponding reduced system (Kreiss, 1980). This system is obtained by noting that the estimates we have derived hold for any value of $\varepsilon$ so that we can consider the limiting case $\varepsilon \rightarrow 0$. To obtain the proper limit, we must ensure that all first-order derivatives are of order unity. As we have seen above, it suffices to ensure that the first-order time derivative is of order unity. This we in effect did by introducing the variable $a$ above. Then we see that the system that the smooth solutions of (2.1) satisfy as $\varepsilon \rightarrow 0$ is

$u_{t}+\bar{u} u_{x}=0$,

$v_{x}=0$,

$\frac{\partial}{\partial t} \int_{0}^{2 \pi} v \mathrm{~d} x=\int_{0}^{2 \pi} f \mathrm{~d} x$

The reduced system is automatically well posed since the energy estimate did not depend on $\varepsilon$. This is also clear from the simple nature of the reduced system.

We can use the reduced system to make asymptotic expansions of the smooth solutions of (2.1). Let the initial conditions for (2.1) be given by

$u(x, 0)=u_{0}(x)$

$v(x, 0)=v_{0}(x)$.

Without loss of generality we can assume that $u_{0}=$ 0 and

$\int_{0}^{2 \pi} v_{0}(x) \mathrm{d} x=\int_{0}^{2 \pi} f(x, t) \mathrm{d} x=0$,

for if this were not the case we would use the smooth solution of the reduced system whose initial conditions are given by

$u(x, 0)=u_{0}(x)$,

$\int_{0}^{2 \pi} v(x, 0) \mathrm{d} x=\int_{0}^{2 \pi} v_{0}(x) \mathrm{d} x$,

as the first term of the asymptotic series. We now choose for the reduced system the initial conditions

$u(x, 0)=0$,

add the forcing term $\varepsilon \bar{v}^{-1} f$ to the right-hand side of the equation (2.5b) for $v$, and replace $(2.5 \mathrm{c})$ with the specification that the mean of $v$ be zero. The unique solution of the reduced system we denote by $u^{0}=0$ and $v^{0}=0(\varepsilon)$. Let us now define the new variables

$u^{d}=u-u^{0}=u$

$v^{d}=v-v^{0}$ 
Solving these two equations for $u$ and $v$ and then substituting into (2.1) we obtain the system

$u_{t}^{d}+\bar{u} u_{x}^{d}=0$,

$v_{t}^{d}+\varepsilon^{-1} \bar{v} v_{x}^{d}=-v_{t}^{0}$,

with the initial conditions

$u^{d}(x, 0)=0$,

$v^{d}(x, 0)=v_{0}(x)-v^{0}(x, 0)$.

Notice that we have reduced the forcing term in (2.1) from order unity to order $\varepsilon$. If we choose $v^{d}(x, 0)=0$, then by Duhamel's principle we know the the norm of $\left(u^{d}, v^{d}\right)^{T}$ is of order $\varepsilon$ so that the solution of (2.1) and the solution $\left(u^{0}, v^{0}\right)^{r}$ of the reduced system will be the same to within an error term of order $\varepsilon$ for a time interval of order unity. We note that by setting $v_{d}(x, 0)=0$ we have chosen for $v$ the initial condition $v_{0}(x)=v^{0}(x, 0)$ which satisfies the initialization constraint (2.4) with $b=0$. We can also repeat this process indefinitely obtaining error terms as small as we wish if the initial data for $v$ is chosen appropriately. At each step the data will satisfy the corresponding initialization constraint derived by the bounded derivative method.

We now consider the corresponding initialboundary value problem, i.e. we consider system (2.1) in the interval $0 \leqslant x \leqslant 1$ with $0 \leqslant t<\infty$. We will assume $\bar{u}<0$ and $\bar{v}>0$ and consider the well-posed boundary conditions for (2.1)

$$
\begin{aligned}
& u(1, t)=r v(1, t)+g(t), \\
& v(0, t)=s u(0, t)+h(t),
\end{aligned}
$$

where $r$ and $s$ are constants of order unity and $g$ and $h$ are known smooth functions. Without loss of generality we can assume $g=h=0$. For if this were not the case then we would define the new variables

$u^{\prime}=u-\tilde{u}$,

$v^{\prime}=v-\tilde{v}$,

where $\tilde{u}$ and $\tilde{v}$ are solutions of what we surmise is the reduced system for the initial-boundary value problem:

$u_{t}+\bar{u} u_{x}=0$

$v_{x}=0$, with the initial condition for $u$ that of the full system and with the boundary conditions

$$
\begin{aligned}
& u(1, t)=r v(1, t)+g(t), \\
& v(0, t)=s u(0, t)+h(t) .
\end{aligned}
$$

Clearly this is a well-posed system with smooth solutions. Solving the expressions defining the primed variables for $u$ and $v$ and then substituting into (2.1) we obtain an equation for $\left(u^{\prime}, v^{\prime}\right)^{T}$ of the same form as (2.1) but with a forcing term of order unity which we can assume is zero without loss of generality. The boundary conditions for this system have the same form as (2.7) with $g=h=0$.

Multiplying the homogeneous version of (2.1) by $Z^{T}$ and then integrating with respect to $x$ from 0 to 1 we obtain the equality

$$
\left.\frac{\partial}{\partial t}\|Z(x, t)\|^{2}=-\mid \bar{u} u(x, t)^{2}+\varepsilon^{-1} \bar{v} v(x, t)^{2}\right\}_{x=0}^{x=1}
$$

A bound of the desired form exists if and only if the right-hand side of (2.10) in conjunction with the homogeneous versions of $(2.7 \mathrm{a})$ and $(2.7 \mathrm{~b})$ is nonpositive. Thus we must choose $r$ and $s$ so that $\bar{u}+\varepsilon^{-1} s^{2} \bar{v} \leqslant 0$ and $r^{2} \bar{u}+\varepsilon^{-1} \bar{v} \geqslant 0$. As in the toroidal problem, we can differentiate (2.1) with respect to $t$ an appropriate number of times to obtain an equation for any time derivative of $Z$. To obtain boundary conditions for these problems, we also differentiate (2.7) exactly as we differentiated (2.1). Then the corresponding time derivative of $Z$ satisfies the same equation and boundary conditions as $Z$ itself (with $f, g$, and $h$ replaced by the corresponding time derivative of $f, g$, and $h$, respectively) so that, as before, if the norm of any time derivative of $Z$ is initially of order unity, then it will be of order unity for all time. Notice that the smoothness of $g$ and $h$ are required here in order that the inhomogeneous terms of the boundary conditions obtained by differentiating (2.7a) and (2.7b) with respect to time remain of order unity. As in the toroidal problem we can estimate spatial derivatives in terms of time derivatives.

From the arguments above we know that as $\varepsilon \rightarrow$ 0 the initial-boundary value problem approaches a reduced initial-boundary value problem which is necessarily well posed. By letting $\varepsilon \rightarrow 0$ in (2.1) and (2.7) we see that the reduced system is in fact given 
by $(2.8)$ and (2.9). Note that the reduced problem has an infinite signal speed, i.e. the boundary data at $x=0$ affects the entire region instantaneously because of the form of the equation for $v$. Then necessarily system (2.1) will also display this behavior for small $\varepsilon$.

In more general initial-boundary value problems the direct approach taken above can be complicated so we illustrate an alternative one. In this approach we surmise that the reduced initialboundary value problem is given by (2.8) and (2.9). If we can prove the well posedness of the reduced system candidate, then its solutions will be smooth since there are no factors of $\varepsilon$ in the reduced system. For our simple example, the well posedness is obvious and in practical problems is easy to prove (see Section 3). We now show that if the initial data for the original initial-boundary value problem is chosen correctly, then the ensuing solution is as close to a solution of (2.8)-(2.9) as we wish.

We assume that the initial conditions for the original initial-boundary value problem are given as

$$
u(x, 0)=u_{0}(x)
$$

$v(x, 0)=v_{0}(x)$

As we have seen before, we can assume $u^{0}=0$ and $g=h=0$. We now solve the reduced system with trivial initial conditions for $u$ and add the inhomogeneous term $\varepsilon \bar{v}^{-1} f$ to the right-hand side of (2.8b). We call this solution $\left(u^{0}, v^{0}\right)^{T}$ and note that the norm of this solution is of order $\varepsilon$. Introducing the change of variables (2.6) into system (2.1) with boundary conditions $(2.7)$, we see that the inhomogeneous function corresponding to $f$ is of order $\varepsilon$. Then if we choose $v^{d}(x, 0)=0$, the norm of $\left(u^{d}\right.$, $\left.v^{d}\right)^{T}$ is of order $\varepsilon$ so that the solution of the original problem is close (to within an error term of order $\varepsilon$ ) to a smooth solution. Clearly this process can be repeated so that the error term can be made as small as one desires. Incidentally we have now proven that $(2.8)-(2.9)$ is in fact the correct reduced initial-boundary value problem.

The implication of these arguments for the initial-boundary value problem is that we need only require that the initial time derivatives of $Z$ be of order unity and that $g$ and $h$ be smooth in time in order for the solution to be smooth in time. The spatial variation of $Z$ is automatically guaranteed to be smooth. Many times in practice $g$ and $h$ are not correctly known and are specified in some $a d$ hoc manner, e.g. they are fixed to be their initial value. Since in this case $g$ and $h$ are certainly smooth in time, the solution will be smooth in time and space. Unfortunately the error on the boundary propagates into the interior at the speed associated with the fast time scale. We will illustrate this difficulty with a numerical example in Section 5 .

It is crucial to recognize that the differentiated versions of the open boundary conditions (2.7) supplied the boundary conditions that were needed for the equations for the time derivatives of $Z$ in the preceding proof. We will now show how to use this fact to ensure that the initial time derivatives are of order unity. As in the toroidal problem, we are free to specify the initial values of $u$. However we must choose the initial values of $v$ so that $v_{x}=\varepsilon a(x)$ where $a$ is a given function whose norm is of order unity. We integrate this equality from 0 to $x$ and find that

$v(x, 0)=s u(0,0)+h(0)+\varepsilon \int_{0}^{x} a(\tilde{x}) \mathrm{d} \tilde{x}$,

where we have replaced $v(0,0)$ using $(2.7 \mathrm{~b})$ at time $t=0$. If $r=0$ then we assume that $u(1,0)=g(0)$ and we are finished. If $r \neq 0$, then from (2.7a) and (2.12) we see that $a$ must satisfy the compatibility constraint

$$
\begin{aligned}
v(1,0) & =s u(0,0)+h(0)+\varepsilon b_{b}^{1} a(\tilde{x}) \mathrm{d} \tilde{x} \\
& =r^{-1}[u(1,0)-g(0)] .
\end{aligned}
$$

The first-order time derivative of $Z$ satisfies the same differential equation and boundary condition as $Z$ (except with $f, g$, and $h$ replaced by $f_{t}, g_{t}$, and $h_{t}$, respectively) and we can use the method above to determine $v_{t}(x, 0)$. Then we can use the differential equation for $v$ to determine $a(x)$ since $a$ $=\varepsilon^{-1} v_{x}=-\bar{v}^{-1} v_{t}$. Thus we have ensured that both the first and second-order time derivatives are of order unity. Clearly this process can be continued indefinitely.

Although the open boundary example we have chosen for this section is very simple, the techniques used to prove the existence of smooth solutions and to derive the corresponding initialization methods can be generalized to more complicated linear and nonlinear hyperbolic systems. In the next section we will apply the concepts to the nonlinear shallow water equations. 
3. Existence of smooth solutions of the shallow water equations for the case of open boundaries

In Cartesian coordinates $x$ and $y$, directed eastward and northward respectively, a dimensionless version of the shallow water equations is (Browning et al., 1980)

$u_{t}+u u_{x}+v u_{y}+\varepsilon^{-1}\left[\phi_{x}-\left(f_{0}+\varepsilon \beta y\right) v\right]=0$,

$v_{z}+u v_{x}+v v_{y}+\varepsilon^{-1}\left[\phi_{y}+\left(f_{0}+\varepsilon \beta y\right) u\right]=0$,

$\phi_{t}+u \phi_{x}+v \phi_{y}+\varepsilon^{-2}\left(\phi_{0}+\varepsilon^{2} \phi\right)\left(u_{x}+v_{y}\right)=0$,

where the variables and parameters are dimensionless with $t$ being time, $u$ and $v$ the velocity components in the $x$ and $y$ directions, $\phi$ a geopotential corresponding to the deviation of the free surface height from its mean, $\varepsilon=10^{-1}, \phi_{0}=1$, $f_{0}=\sin \theta_{0}, \beta=\cos \theta_{0}$, and $\theta_{0}$ the latitude of the coordinate origin. To simplify the presentation we will assume that $\beta=0$. We consider system (3.1) in the rectangle $R=\{(x, y): 0 \leqslant x \leqslant 1,0 \leqslant y \leqslant 2 \pi\}$ with initial conditions

$u(x, y, 0)=\bar{u}(x, y)$,

$v(x, y, 0)=\dot{v}(x, y)$,

$\phi(x, y, 0)=\dot{\phi}(x, y)$,

and with periodic boundary conditions in the $y$ direction. We will discuss suitable boundary conditions at $x=0$ and $x=1$ in the following discussion.

Linearizing (3.1) about the initial data, we can write the variational system in the form (Browning et al., 1980)

$\left(\frac{\partial}{\partial t}+A_{1} \frac{\partial}{\partial x}+A_{2} \frac{\partial}{\partial y}+A_{0}\right) Z=0$,

where

$A_{1}=\left[\begin{array}{ccc}\bar{u} & 0 & \varepsilon^{-3 / 2} d \\ 0 & \bar{u} & 0 \\ \varepsilon^{-3 / 2} d & 0 & \bar{u}\end{array}\right], \quad A_{2}=\left[\begin{array}{ccc}\bar{v} & 0 & 0 \\ 0 & \bar{v} & \varepsilon^{-3 / 2} d \\ 0 & \varepsilon^{-3 / 2} d & \bar{v}\end{array}\right]$

$A_{0}=\left[\begin{array}{ccc}\bar{u}_{x} & -\varepsilon^{-1} f_{0}-\beta y+\bar{u}_{y} & \varepsilon^{-3 / 2} d_{x} \\ \varepsilon^{-1} f_{0}+\beta y+\bar{v}_{x} & \bar{v}_{y} & \varepsilon^{-3 / 2} d_{y} \\ \varepsilon^{1 / 2} d^{-1} \bar{\phi}_{x} & \varepsilon^{-1 / 2} d^{-1} \bar{\phi}_{y} & d^{-1}\left(d_{x} \bar{u}+d_{y} \bar{v}\right)+\left(\bar{u}_{x}+\bar{v}_{y}\right)\end{array}\right] . \quad Z=\left[\begin{array}{c}u \\ v \\ \varepsilon^{1 / 2} d^{-1} \phi\end{array}\right]$.

Tellus 34 (1982), 4 and $d=\left(\phi_{0}+\varepsilon^{2} \phi\right)^{1 / 2}$. We have used the same symbols for the perturbation quantities as those of (3.1) to simplify the notation and, without loss of generality, have dropped forcing terms of order unity. Now that the large part of the operator has been symmetrized, lower order terms with coefficients of order unity can be ignored without restriction since they only lead to a slow growth rate of the norm of the solution. Since the partial derivatives of $d$ are of order $\varepsilon^{2}$, the only possible large terms of $A_{0}$ are the Coriolis terms $\pm \varepsilon^{-1} f_{0}$ and we can ignore the remaining terms. Then there are two cases to consider: either $f_{0}=0$ or $f_{0} \neq 0$.

We first consider the equatorial case, i.e. we assume $f_{0}=0$ so that in this case we can assume $A_{0}$ $=0$. Kreiss (1980) has proven a general theorem about the existence of smooth solutions of symmetric hyperbolic systems with multiple time scales. We see that for the equatorial case (3.3) is in the second normal form that he derived so that his results are directly applicable. We state the theorem he proved in terms of our special case:

\section{Theorem 3.1}

Consider the inhomogeneous problem

$\left(\frac{\partial}{\partial t}+A_{1} \frac{\partial}{\partial x}+A_{2} \frac{\partial}{\partial y}\right) Z=F$

where

$F=\left[\begin{array}{c}F_{1} \\ F_{2} \\ \varepsilon^{-3 / 2} F_{3}\end{array}\right]$

with initial data (3.2) such that $\dot{u}(0, y)>0$ and $\bar{u}(1, y)>0$ and boundary data

$\phi(0, y, t)=-\varepsilon r_{0} u(0, y, t)+\varepsilon g_{0}(y, t)$,

$v(0, y, t)=h(y, t)$,

$\phi(1, y, t)=\varepsilon r_{1} u(1, y, t)+\varepsilon g_{1}(y, t)$. 
Assume that all the data are smooth functions, that the initial conditions are compatible with the boundary conditions, and that at $t=0$ a couple of time derivatives are of order unity. Then $u, v$, and $\delta$ $=\varepsilon^{-1} \phi$ converge for $\varepsilon \rightarrow 0$ to a smooth solution of the reduced equation

$u_{t}+\bar{u} u_{x}+\bar{v} u_{y}+\tilde{\phi}_{x}=F_{1}$,

$v_{t}+\bar{u} v_{x}+\bar{v} v_{y}+\bar{\phi}_{y}=F_{2}$,

$u_{x}+v_{y}=F_{3}$,

with initial data

$u(x, 0)=\bar{u}(x, y)$,

$v(x, 0)=\bar{v}(x, y)$,

and boundary conditions

$\tilde{\phi}(0, y, t)=-r_{0} u(0, y, t)+g_{0}(y, t)$,

$v(0, y, t)=h(y, t)$,

$\bar{\phi}(1, y, t)=r_{1} u(1, y, t)+g_{1}(y, t)$.

Kreiss assumed that $F_{3}=0$. If $F_{3} \neq 0$ then we can subtract from $Z$ the function

$Z^{\prime}=\left[\begin{array}{c}\int_{0}^{x} F_{3}(\tilde{x}, y) \mathrm{d} \tilde{x} \\ 0 \\ 0\end{array}\right]$.

The difference $Z^{d}=Z-Z^{\prime}$ satisfies (3.4) with $F_{3}=$ 0 and $F_{1}$ and $F_{2}$ replaced by the appropriate functions. The boundary conditions (3.5a) and (3.5c) state that on the boundaries $\phi$ is of order $\varepsilon$. This is physically reasonable since we know that $\phi$ is of order $\varepsilon$ near the equator. Although Kreiss did not have any $\varepsilon$ factors in $Z$, this does not pose any difficulty. Once we know that the reduced system (3.6)-(3.8) is well posed, we can expand the smooth solutions of (3.3) in terms of solutions of the reduced system. Since we will show how to do this for the midlatitudes, we will not go into detail here as it is essentially the same procedure.

We now briefly review the proof of Theorem 3.1 to point out the salient results. Multiplying (3.4) by $Z^{T}$ and integrating over the region $R$ we have

$$
\begin{aligned}
& \frac{\partial}{\partial t}\|Z\|^{2}=-\int_{0}^{2 \pi}\left[Z^{T} A_{1} Z\right]_{0}^{1} \mathrm{~d} y \\
& \quad-\int_{0}^{1}\left[Z^{T} A_{2} Z\right]_{0}^{2 \pi} \mathrm{d} x+2 \int_{R} Z^{T} E Z \mathrm{~d} x \mathrm{~d} y,
\end{aligned}
$$

where $2 E=\partial A_{1} / \partial x+\partial A_{2} / \partial y$. The second term on the right-hand side of (3.9) is zero by the assumption of periodicity in the $y$ direction. Since $\|E\|=0(1)$, without loss of generality we can assume $E=0$. Then we can rewrite (3.9) as

$$
\begin{aligned}
\frac{\partial}{\partial t} & \|Z\|^{2} \\
= & -\int_{0}^{2 \pi}\left[\bar{u}\left(u^{2}+v^{2}+\varepsilon d^{-2} \phi^{2}\right)+\left.2 \varepsilon^{-1} u \phi\right|_{0} ^{1} \mathrm{~d} y\right. \\
= & -\int_{0}^{2 \pi}\left[\bar{u} v^{2}+.5\left(\bar{u}+\varepsilon^{-3 / 2} d\right)\left(u+\varepsilon^{1 / 2} d^{-1} \phi\right)^{2}\right. \\
& \left.+.5\left(\bar{u}-\varepsilon^{-3 / 2} d\right)\left(u-\varepsilon^{1 / 2} d^{-1} \phi\right)^{2}\right]_{0}^{1} \mathrm{~d} y,
\end{aligned}
$$

where in the second step we have introduced the one-dimensional characteristic variables following the derivation of Oliger and Sundström (1978). Since we have assumed that $\bar{u}(0, y)>0$ (inflow) we see from (3.10) that at $x=0$ there are two incoming characteristics so that the number of boundary conditions at $x=0$ is correct. Since $\bar{u}(1$, y) $>0$ (outflow), at $x=1$ there is only one incoming characteristic and again the number of boundary conditions is correct. Substituting the homogeneous versions of (3.5) into the right-hand side of (3.10) we see that the necessary condition of nonpositivity is satisfied if $r_{0}$ and $r_{1}$ are within a certain range which we will discuss later. Without loss of generality we can assume that $g_{0}=g_{1}=h=$ 0 so that this estimate is all that we need. For if this were not the case, then we could define the new variable $Z^{d}=Z-Z^{\prime}$ where $Z^{\prime}(x, y, t)$ satisfies the requirements

$$
\begin{aligned}
& \phi^{\prime}(0, y, t)=-\varepsilon r_{0} u^{\prime}(0, y, t)+\varepsilon g_{0}(y, t), \\
& v^{\prime}(0, y, t)=h(y, t), \\
& \phi^{\prime}(1, y, t)=\varepsilon r_{1} u^{\prime}(1, y, t)+\varepsilon g_{1}(y, t), \\
& \phi_{x}^{\prime}=\varepsilon a(x, y, t), \\
& \phi_{y}^{\prime}=\varepsilon b(x, y, t), \\
& u_{x}^{\prime}+v_{y}^{\prime}=\varepsilon^{2} c(x, y, t),
\end{aligned}
$$

where $a, b$, and $c$ are given smooth functions and satisfy the constraint

$-a_{y}+b_{x}=0$.

The requirements $(3.11 \mathrm{a}-\mathrm{c})$ are the standard ones to ensure that $Z^{d}$ will satisfy (3.5) with homogeneous boundary data, whereas the requirements (3.11d-f) ensure that the forcing term in the equation for $Z^{d}$ will be of order unity. Equation 
(3.11g) is a compatibility constraint (Browning et al., 1980).

To show that such a $Z^{\prime}$ exists, we consider two cases. If the boundary data are obtained from the smooth solution $Z_{C}$ of the related Cauchy problem, i.e. from the solution of (3.4) in a rectangle $R_{C}=$ $\left\{(x, y): x_{0} \leqslant x \leqslant x_{1}, 0 \leqslant y \leqslant 2 \pi, x_{0} \leqslant 0, x_{1} \geqslant 1\right\}$ with properly extended versions of (3.2) for initial conditions and with periodic boundary conditions in both $x$ and $y$, then we can simply let $Z^{\prime}=Z_{c}$. Since $Z_{C}$ is a slow scale solution of the related Cauchy problem, $Z^{\prime}$ must satisfy $(3.11 \mathrm{~d}-\mathrm{g})$ and, by assumption, it also satisfies $(3.11 \mathrm{a}-\mathrm{c})$. In fact in this case there will be no forcing term in the equation for $Z^{d}$ since $Z^{\prime}$ is a solution of system (3.4). From the nonpositivity of the right-hand side of $(3.10)$ we have that

$\left\|Z^{d}(x, y, t)\right\| \leqslant\left\|Z^{d}(x, y, 0)\right\|=0$.

Of course this just means that we can exactly duplicate the solution of the Cauchy problem in the region $R$ if we first run the related Cauchy problem to obtain the boundary conditions we need. Note that in this case the balanced initial data for (3.4) in the rectangle $R$ are identical to the proper restriction of the balanced initial values for the related Cauchy problem. However, in Section 4 we will show that the initial values for $R$ can be computed without reference to the related Cauchy problem.

The more interesting case is when we do not want to run the related Cauchy problem. Then the boundary data will normally be incorrect and the errors on the boundary of $R$ will inevitably destroy the solution in the interior. Although we will show that the boundary data can be specified so that the solution will be smooth, any errors in the data will propagate into the interior of $R$ at the speed associated with the fast time scale, e.g. in Section 5 we demonstrate that errors in the boundary data destroy the geopotential in a matter of hours and so must be kept small if the solution in the interior is to be computed correctly. As in Section 2 we show that in this case we only need to require that the initial time derivatives be of order unity and that the boundary data be smooth in order to guarantee that the solution will be smooth. In this case we choose for $Z^{\prime}$ the values

$\left[\begin{array}{c}u^{\prime} \\ v^{\prime} \\ \varepsilon^{1 / 2} d^{-1} \phi^{\prime}\end{array}\right]=\left[\begin{array}{c}-(2 \pi)^{-1} h_{y} \sin 2 \pi x \\ h \cos 2 \pi x \\ \varepsilon^{3 / 2} d^{-1}\left[(1-x) g_{0}+x g_{1}\right]\end{array}\right]$, which is smooth since the boundary data were assumed to be smooth. That $Z^{\prime}$ satisfies (3.11) (with $c=0$ ) can be verified by substitution. Again we have the estimate $\left\|Z^{d}(x, y, t)\right\| \leqslant\left\|Z^{d}(x, y, 0)\right\|$ for the homogeneous equation and boundary conditions for $Z^{d}$. In this case the forcing term in the equation for $Z^{d}$ is not zero, but is of order unity so that without restriction it can be ignored.

As in Section 2 we note that any time derivative of $Z$ satisfies the same equations and boundary conditions as $Z$ (except with the boundary data replaced by their corresponding time derivative) so that the above arguments also apply to any time derivative of $Z$. We can also differentiate the equations and boundary conditions with respect to $y$, but lower order terms involving derivatives with respect to $x$ appear in this process because of the variable coefficients. Without restriction we assume that $\bar{u}(x, y)>0$ so that we can solve (3.4) for $Z_{x}$ in terms of $Z_{t}$ and $Z_{y}$. We can write the first and last equations of (3.4) in the form

$\left[\begin{array}{cc}\bar{u} & \varepsilon^{-1} \\ \varepsilon^{-2} d^{2} & \bar{u}\end{array}\right]\left[\begin{array}{l}u_{x} \\ \phi_{x}\end{array}\right]=-\left[\begin{array}{c}u_{t}+\bar{v} u_{y} \\ \phi_{t}+\bar{v} \phi_{y}+\varepsilon^{-2} d^{2} v_{y}\end{array}\right]$

Each of the components of the solution of this system is expressed as a linear combination of the components of $Z, Z_{t}$, and $Z_{y}$ with coefficients of order unity. Thus we can use these relations to replace $u_{x}$ and $\phi_{x}$ in the equation for the $y$ derivative of $Z$. However, when we solve the second equation of (3.4) for $v_{x}$ in the form

$\bar{u} v_{x}=-\left(v_{t}+\bar{v} v_{y}+\varepsilon^{-1} \phi_{y}\right)$,

we find that the linear combination involves a constant of order $\varepsilon^{-1}$ and so it appears that we cannot use expression (3.13) to replace $v_{x}$ in the equation for the $y$ derivative of $Z$. Equation (3.11g) implies that the vector $(a, b)^{T}$ is the gradient of a scalar function, e.g. $(a, b)^{7}=\nabla \bar{\phi}$. This suggests the substitution $\phi=\varepsilon \tilde{\phi}$ which is reasonable from physical grounds since we know that $\phi$ is or order $\varepsilon$ at the equator. We make this change of variables in (3.13). This would then allow us to use (3.13) to replace $v_{x}$ if we ensure that $\tilde{\phi}$ and $\tilde{\phi}_{y}$ are of order unity. The introduction of $\tilde{\phi}$ is equivalent to bounding the first-order time derivatives of $u$ and $v$. From Section 2 we saw that at this step we could say nothing about the actual form of $\bar{\phi}$. But from the results of Section 2 we expect to obtain

Tellus 34 (1982), 4 
additional information about $\bar{\phi}$ by proceeding to the second-order time derivative. From previous work we know that the time derivative of the divergence enters as an initialization constraint in the secondorder time derivative so we form the divergence equation

$\delta_{t}+\bar{v} \delta_{x}+\bar{v} \delta_{y}+\bar{\delta} \delta-J+\nabla^{2} \bar{\phi}=0$

where $\delta=u_{x}+v_{y}$ and $J=\bar{u}_{x} v_{y}+u_{x} \bar{v}_{y}-\bar{u}_{y} v_{x}$ $u_{y} \bar{v}_{x}$. This equation coupled with the homogeneous versions of (3.5a) and (3.5c) provides estimates of $\bar{\phi}$ and $\tilde{\phi}_{y}$ of the forms (Kreiss, 1980)

$$
\begin{aligned}
& \|\bar{\phi}\| \leqslant C_{1}\left(\|Z\|+\left\|Z_{t}\right\|+|u|_{b}\right)+0\left(\varepsilon^{2}\right), \\
& \left\|\tilde{\phi}_{y}\right\| \leqslant C_{2}\left(\|Z\|+\left\|Z_{t}\right\|+\left\|Z_{y}\right\|+|u|_{b}+\left|u_{y}\right|_{b}\right) \\
& \quad+0\left(\varepsilon^{2}\right),
\end{aligned}
$$

where $|u|_{b}^{2}=\left.\left.\int_{0}^{2 \pi}|| u\right|^{2}\right|_{0} ^{1} \mathrm{~d} y$ and $C_{1}$ and $C_{2}$ are constants. It is now possible to estimate the $y$ derivatives and for the details we refer the reader to Kreiss' paper. Estimates of the $x$ derivatives follow from the linear combinations in (3.12) and (3.13).

Since the bounds we have derived are independent of $\varepsilon$, as in Section 2 we can let $\varepsilon \rightarrow 0$ to obtain the reduced initial-boundary value problem (3.6)(3.8) and this system must be well posed. We can differentiate (3.6c) (with $F_{3}=0$ ) with respect to $t$ yielding

$\nabla^{2} \tilde{\phi}=J$,

and in this form the reduced problem can be shown to be well posed independent of the limiting argument (Gustafsson and Kreiss, 1980). For very small $\varepsilon$, extreme accuracy is needed to compute the smooth solutions of the original system due to the necessary cancellation of terms. In such cases the reduced system is preferred as no such cancellation occurs and the reduced system describes the smooth solution very accurately. As in Section 2 we see that the reduced system has an infinite signal speed due to the elliptic operator. Any errors in the pressure data on the boundary will be spread throughout the region instantaneously. Again we must keep the errors on the boundary small if the solution in the interior is to be computed correctly. However here we only require that the error in the large-scale boundary data be small, i.e. large errors in the small-scale boundary data are damped exponentially as a function of the distance from the boundary.
We shall now generalize the boundary conditions (3.5) slightly. For any $s>0$ the estimates which lead to Theorem 3.1 do not depend on $r_{0}$ and $r_{1}$ provided

$0.5 \bar{u}+s \leqslant r_{0} \leqslant 2 \varepsilon^{-3} d^{2} \bar{u}^{-1}-s \quad(x=0)$,

$r_{1} \geqslant-0.5 \vec{u}+s \quad(x=1)$.

Thus we can consider the case that $\lim _{\varepsilon \rightarrow 0} r_{0}=\infty$ or $r_{1}=\infty$ or both. For the reduced system the boundary conditions become

$u(0, y, t)=0 \quad$ or $\quad u(1, y, t)=0$

or

$u(0, y, t)=0 \quad$ and $\quad u(1, y, t)=0$.

It is also possible to consider inhomogeneous boundary data for the reduced system. Corresponding to (3.17) we can consider

$u(0, y, t)=g_{0}(y, t) \quad$ or $\quad u(1, y, t)=g_{1}(y, t)$.

To show this we consider boundary conditions for the reduced system of the form

$\bar{\phi}(0, y, t)=-r_{0}\left(u(0, y, t)-g_{0}(y, t)\right)$,

$v(0, y, t)=h(y, t)$,

$\delta(1, y, t)=r_{1}\left(u(1, y, t)-g_{1}(y, t)\right)$.

Making the change of variables

$u^{\prime}=u-g_{0}(y, t)-\int_{0}^{x} F_{3}(\tilde{x}, y) \mathrm{d} \tilde{x}$

or

$u^{\prime}=u-g_{1}(y, t)-\int_{0}^{x} F_{3}(\tilde{x}, y) \mathrm{d} \tilde{x}$,

we transform the reduced problem to the previous case. The full system transforms to a system of the same form as before. We can use this same procedure for inhomogeneous boundary conditions corresponding to (3.18) only if the compatibility condition

$\int_{0}^{1}\left(g_{1}-g_{0}\right) \mathrm{d} y=\int_{0}^{1} \int_{0}^{2 \pi} F_{3} \mathrm{~d} x \mathrm{~d} y$,

is satisfied. This condition is necessary as can be seen by integrating (3.6c). It is also sufficient. We define the functions $u^{\prime}, v^{\prime}$, and $\phi^{\prime}$ by

$$
\begin{aligned}
u^{\prime} & =\int_{0}^{x} F_{3}(\tilde{x}, y) \mathrm{d} \tilde{x}+(1-x) g_{0}(y) \\
& -x \int_{0}^{1} F_{3}(\tilde{x}, y) \mathrm{d} \tilde{x}+x g_{1}(y), \\
v^{\prime} & =-\int_{0}^{y}\left[g_{1}(\tilde{y})-g_{0}(\tilde{y})-\int_{0}^{1} F_{3}(\tilde{x}, \tilde{y}) \mathrm{d} \tilde{x}\right] \mathrm{d} \tilde{y}, \\
\phi^{\prime} & =0 .
\end{aligned}
$$


The functions $u^{\prime}$ and $\phi^{\prime}$ are clearly periodic in $y$ and by (3.20) so is $v^{\prime}$. The difference $Z^{d}=Z-Z^{\prime}$ satisfies (3.4) with $F_{3}=0$ and boundary conditions (3.5) with $g_{0}=g_{1}=h=0$. Thus we can also expand the solutions of (3.4) with the boundary conditions (3.19) in terms of the reduced problem and, in particular, the values $\lim _{\varepsilon \rightarrow 0} r_{0}=\infty$ or $r_{1}=\infty$ or both are permissible.

In the midlatitudes $f_{0} \neq 0$ and by arguments similar to those described above it suffices to consider the system

$$
\left(\frac{\partial}{\partial t}+A_{1} \frac{\partial}{\partial x}+A_{2} \frac{\partial}{\partial y}+A_{0}\right) Z=F,
$$

where $A_{1}$ and $A_{2}$ are as in (3.3.),

$A_{0}=\left[\begin{array}{ccc}0 & -\varepsilon^{-1} f_{0} & 0 \\ \varepsilon^{-1} f_{0} & 0 & 0 \\ 0 & 0 & 0\end{array}\right]$,

and

$\boldsymbol{F}=\left[\begin{array}{c}F_{1} \\ F_{2} \\ \varepsilon^{-1 / 2} F_{3}\end{array}\right]$.

The forcing term in the third equation is less than that in (3.4) so that for smooth solutions the divergence is at most of order $\varepsilon$ as required by the geostrophic constraints. There are now lower order terms with coefficients which are not of order unity. The general theory allows such terms if they are in the proper normal form. Unfortunately the Coriolis terms are not in one of these forms. If we proceeded as in the equatorial case, then we would encounter the same difficulty estimating the $x$ derivatives in terms of the $y$ derivatives as before. Then we would again have to make a change of variables to complete the proof. Instead of that approach we examine the general theory and find that it is sufficient to choose boundary conditions in such a way that they

(1) lead to an energy estimate for the solutions of (3.21) and

(2) lead to a well-posed problem for the reduced system.

We adopt this approach and show that by making a change of variables in what we surmise is the reduced system for the midlatitudes

$u_{t}+\bar{u} u_{x}+\bar{v} u_{y}+\varepsilon^{-1}\left(\phi_{x}-f_{0} v\right)=F_{1}$

$v_{t}+\bar{u} v_{x}+\bar{v} v_{y}+\varepsilon^{-1}\left(\phi_{y}+f_{0} u\right)=F_{2}$

$u_{x}+v_{y}=\varepsilon F_{3}$

we can reduce (3.22) (with the proper boundary conditions) to a form of the equatorial reduced system and thus show that it is well posed. Then we will prove the existence of smooth solutions of (3.21) by asymptotic expansion of solutions of (3.21) in terms of solutions of (3.22) and estimate the remainder using (1).

We start with (2), i.e. we investigate what type of boundary conditions lead to a well-posed problem for (3.22). Without restriction we can assume that

$F_{3}=0$.

If not we use the previous procedure to ensure that $F_{3}=0$. At the inflow side we give as boundary conditions

$u(0, y, t)=g_{0}(y, t)$,
$v(0, y, t)=h(y, t)$,

where $g_{0}$ cannot be arbitrary. For smooth solutions the geostrophic relation says that

$\phi_{y}=-f_{0} u+0(\varepsilon)$

or upon integration

$0=\int_{0}^{1} \phi_{y} \mathrm{~d} y=-\int_{0} \int_{0}^{1} u \mathrm{~d} y+\mathrm{d} y+0(\varepsilon)$,

which at $x=0$ becomes

$\int_{0}^{1} g_{0}(y t) \mathrm{d} y=0(\varepsilon)$.

Without restriction we can assume that

$\int_{0}^{1} g_{0}(y, t) \mathrm{d} y=0$.

Otherwise we write

$g_{0}(y, t)=g_{00}(y, t)+\varepsilon g_{01}(y, t), \quad \int_{0}^{1} g_{00}(y, t) \mathrm{d} y=0$,

and replace $u$ in (3.22) by $u+\varepsilon g_{01}(y, t)$. Let $u, v$, and $\phi$ be a solution of (3.22). Integrating the third equation of (3.22) with (3.23) gives us that

$\frac{\mathrm{d}}{\mathrm{d} x} \int_{0}^{1} u(x, y) \mathrm{d} y=-\left.v(x, y)\right|_{\substack{y=1 \\ y=0}=0,}$

which with (3.25) implies that

$\int_{0}^{1} u(x, y) \mathrm{d} y=0$

Tellus 34 (1982), 4 
Therefore the overdetermined system

$$
\begin{aligned}
& \phi_{0 x}=f_{0} v, \\
& \phi_{0 y}=-f_{0} u, \\
& \phi_{0}(1,0)=0,
\end{aligned}
$$

has a unique solution which is periodic in $y$. Letting $\phi=\phi_{0}+\varepsilon \tilde{\phi}$ we see that $u, v$, and $\bar{\phi}$ satisfy the reduced system (3.6) with the boundary conditions (3.24a) and (3.24b). For that system we know that we obtain a well-posed problem with smooth solutions if we specify at $x=1$ the boundary condition

$$
\delta(y, t)=r_{1}\left[u(y, t)-g_{1}(y, t)\right] .
$$

Therefore we also obtain a well-posed problem with smooth solutions for system (3.22) with the boundary conditions (3.24a) and (3.24b) and

$$
\begin{aligned}
\phi(1, y, t)= & -f_{0} \int_{0}^{y} u(1, \tilde{y}, t) \mathrm{d} \tilde{y}+\varepsilon r_{1}[u(y, t) \\
& \left.-g_{1}(y, t)\right] .
\end{aligned}
$$

The most attractive boundary conditions are

$r_{1}=\infty\left[u(y, t)=g_{1}(y, t) ; \int_{0}^{1} g_{1}(y, t) \mathrm{d} y=0\right]$

or

$r_{1}=0$ (geostrophic approximation).

Instead of the boundary condition (3.24a) at $x=$ 0 one could think of using a more general boundary condition of type (3.28). This is possible if we use (3.29) at $x=1$ (outflow). Otherwise it seems to be difficult to ensure that $\int_{0}^{1} u(x, y) \mathrm{d} y=0$. Thus at $x=$ 0 (inflow) we will give $u$.

We now determine boundary conditions for the full system which in the limit approach (3.24) and (3.29) or (3.30) and which lead to an energy estimate of the right form for the corresponding solutions of (3.21). At $x=0$ we choose the boundary conditions

$$
\begin{aligned}
& \phi(0, y, t)=-\varepsilon^{-2} \tilde{r}_{0}\left\{u(0, y, t)-g_{0}(y, t)\right\}, \\
& v(0, y, t)=h(y, t),
\end{aligned}
$$

where $0.5 \varepsilon^{3} \bar{u} \leqslant \tilde{r}_{0} \leqslant 2 d^{2} \bar{u}^{\prime}$ and at $x=1$ the boundary condition

$u(1, y, t)=g_{1}(y, t)$

or

$\phi(1, y, t)=f_{0}\left(\int_{0}^{y} u \mathrm{~d} \tilde{y}-y \int_{0}^{1} u \mathrm{~d} y\right)$.

The matrix $A_{0}$ is skew-symmetric and contributes nothing to the energy estimate so that (3.10) also holds in the midlatitudes. Without restriction we can assume that $g_{0}=0\left(\varepsilon^{4}\right)$ and $g_{1}=h=0$. Otherwise we would subtract off the solution of the corresponding reduced problem. Then we can also assume $g_{0}=0$ as it only leads to a slow growth rate of the norm of the solution. We can use the energy estimate (3.10) to prove the existence of a smooth solution only if the right-hand side in conjunction with the homogeneous version of the boundary conditions is nonpositive. We have chosen $\tilde{r}_{0}$ so that this is true for (3.31) and (3.32). This is also true for (3.31) and (3.33) since integration by parts shows that

$\int_{0}^{1}\left[\left(\int_{0}^{y} u \mathrm{~d} \tilde{y}-y \int_{0}^{1} u \mathrm{~d} \tilde{y}\right)-u(1, y, t)\right] \mathrm{d} y=0$.

The boundary conditions (3.31a) and (3.32) lead to the specification of $u$ on the boundary for the reduced system. When specifying $u$ on a boundary for the reduced system, we derive boundary conditions for (3.15) by solving (3.6a) for $\tilde{\phi}_{x}$ in the form

$\tilde{\phi}_{x}=-\left(u_{t}-\bar{u} v_{y}+\bar{v} u_{y}\right)$,

and on the boundary everything on the right-hand side of this equation is known. If we specify $u$ on both boundaries then it is well known that equation (3.15) with Neumann conditions in $x$ and periodic conditions in $y$ only determines $\phi$ up to a constant. Since the solutions of system (3.3) with the boundary conditions (3.31) and (3.32) are unique, we must be able to derive an equation from the original system that uniquely determines this constant (otherwise this could not be the correct reduced system). To show this we integrate the third equation of (3.1) over $R$ obtaining

$$
\begin{aligned}
& \frac{\partial}{\partial t} \int_{R} \phi \mathrm{d} x \mathrm{~d} y=-\int_{0}^{2 \pi}\left[\bar{u} \phi+\left(\varepsilon^{-2} \phi_{0}+\bar{\phi}\right) u\right]_{0}^{1} \mathrm{~d} y \\
& \quad=-\phi_{0} m-\int_{0}^{2 \pi}\left\{\left[\left(\phi_{0}+\bar{u}+\varepsilon^{2} \bar{\phi}\right) \phi\right]_{0}^{1}+\bar{\phi}(1, y, t) g_{1}\right. \\
& \left.\quad+\bar{\phi}(0, y, t) g_{0}\right\} \mathrm{d} y,
\end{aligned}
$$

where, without restriction for smooth solutions, we have assumed that $\int_{0}^{2 \pi}\left(g_{1}-g_{0}\right) \mathrm{d} y=\varepsilon^{2} m(y, t)$ where $m$ is a smooth function. Formally in the limit as $\varepsilon \rightarrow 0$ this equation approaches

$$
\begin{aligned}
\frac{\partial}{\partial t} \int_{R} \phi \mathrm{d} x \mathrm{~d} y \\
\quad=-\phi_{0} m-\int_{0}^{2 \pi}\left\{\left[\left(\phi_{0}+\bar{u}\right) \phi\right]_{0}^{1}+\bar{\phi}(1, y, t) g_{1}\right. \\
\left.\quad+\bar{\phi}(0, y, t) g_{0}\right\} \mathrm{d} y,
\end{aligned}
$$


and this is the equation we use to determine the mean of $\phi$ for the reduced system.

We now show that smooth solutions of system (3.21) exist by asymptotic expansion of those solutions in terms of solutions of system (3.22). We perform the expansion for the boundary conditions (3.31) and (3.32) and note that the other case can be handled similarly. In the first step we choose for initial data for the reduced system the rotational portion of the initial wind data $\left(u_{0}, v_{0}\right)^{T}$ for the original system and for boundary data the functions $\tilde{g}_{0}, \tilde{g}_{1}$, and $\tilde{h}$ where the tilde denotes the nondivergent portion. We assume $g_{0}-\tilde{g}_{0}=O\left(\varepsilon^{2}\right)$, $g_{1}-\tilde{g}_{1}=0\left(\varepsilon^{2}\right)$, and $h-\tilde{h}=0\left(\varepsilon^{2}\right)$. This is no restriction since we are interested in smooth solutions which must satisfy $u_{x}+v_{y}=O\left(\varepsilon^{2}\right)$. We know that we can obtain a smooth solution of system (3.22) with these initial and boundary data from the arguments above. Let us call this solution $Z^{0}$. Then we define a new variable $Z^{d}=Z-Z^{0}$. Solving this expression for $Z$ and substituting into system (3.21), we get for $Z^{d}$ the problem

$$
\begin{aligned}
& u_{t}+\bar{u} u_{x}+\bar{v} u_{y}+\varepsilon^{-1}\left(\phi_{x}-f_{0} v\right)=0 \\
& v_{t}+\bar{u} v_{x}+\bar{v} v_{y}+\varepsilon^{-1}\left(\phi_{y}+f_{0} u\right)=0 \\
& \phi_{t}+\bar{u} \phi_{x}+\bar{v} \phi_{y}+\varepsilon^{-2} d^{2}\left(u_{x}+v_{y}\right) \\
& \quad=-\left(\phi_{t}^{0}+\bar{u} \phi_{x}^{0}+\bar{v} \phi_{y}^{0}\right)
\end{aligned}
$$

with boundary conditions

$$
u(0, y, t)=-\varepsilon^{2} \phi(0, y, t)-\varepsilon^{2} \phi^{0}(0, y, t)+g_{0}-\tilde{g_{0}}
$$

$v(0, y, t)=h(y, t)-\hbar(y, t)$,

$u(1, y, t)=g_{1}-\tilde{g}_{1}$,

where we have dropped the superscript $d$ to simplify the notation. Note that we have added a forcing term of order unity while the inhomogeneous boundary data terms have been reduced to order $\varepsilon^{2}$. We now write $(3.34 c)$ in the form

$$
\begin{gathered}
u_{x}+v_{y}+\varepsilon^{2} d^{-2}\left(\phi_{t}+\bar{u} \phi_{x}+\bar{v} \phi_{y}\right) \\
=-\varepsilon^{2} d^{-2}\left(\phi_{t}^{0}+\bar{u} \phi_{x}^{0}+\bar{v} \phi_{y}^{0}\right) .
\end{gathered}
$$

We now solve system (3.22) with trivial initial and boundary data and the forcing term in (3.35) for the right-hand side of $(3.22 \mathrm{c})$. We call this solution $Z^{1}$ and note that its norm is of order $\varepsilon^{2}$. Then in the equation for $Z^{d}=Z-Z^{0}-Z^{1}$ the boundary data terms and the forcing term are of order $\varepsilon^{2}$. Then if we choose $Z^{d}(x, y, 0)=0$, the energy estimate (3.5) tells us that the norm of $Z^{d}$ is or order $\varepsilon$. Thus if we choose the initial data for $Z$ correctly, then $Z$ will be close (to within an error term of order $\varepsilon$ ) to a smooth solution for any time interval of order unity. This also tells us that (3.22) is the reduced system since as $\varepsilon \rightarrow 0, Z^{d} \rightarrow 0$ and $Z^{1} \rightarrow 0$. Clearly we can repeat this process indefinitely obtaining error terms as small as we wish. Then if the initial data for the original system is chosen correctly, it will have smooth solutions up to order $\varepsilon^{n}$ with $n$ as large as we wish.

In ensuing steps of the iteration to prove the existence of smooth solutions of system (3.1), we must linearize about the previous solution so that the overbar quantities of (3.2) also become functions of $t$. Although the proof for these steps is more complicated than the one above (Kreiss, 1980), essentially no new information is added. For practical applications these arguments have important implications. If we wish to generate solutions which are smooth in time and space, we must ensure that not only the initial time derivatives be of order unity, but also that the boundary data be smooth. In addition the errors on the boundary will always propagate into the interior at the rate associated with the fast time scale and therefore must be kept small. We will employ these concepts in the numerical examples of Section 5.

\section{Initialization of the shallow water equa- tions for the case of open boundaries}

In a previous paper (Browning et al., 1980) we derived initialization constraints for the shallow water equations with an equivalent depth corresponding to the external mode of the atmosphere (Williamson and Dickinson, 1976). However, only periodic and solid wall boundary conditions were treated. In this section we treat the case of open boundaries. We will only treat the midlatitude case as the equatorial case has already been treated (Kreiss, 1980). Thus we will consider system (3.1) in the region $R$ of Section 3 with $f_{0} \neq 0$.

The first-order time derivative of system (3.1) is of order unity if and only if

$\phi_{x}-f_{0} v=\varepsilon a(x, y, t)$,
$\phi_{y}+f_{0} u=\varepsilon b(x, y, t)$,
$u_{x}+v_{y}=\varepsilon^{2} c(x, y, t)$,

Tellus 34 (1982), 4 
where $a, b$, and $c$ are smooth functions which satisfy

$-a_{y}+b_{x}=\varepsilon f_{0} c$.

The earliest initialization schemes assumed geostrophic balance i.e they assumed $a=b=c=0$ at $t=0$. Then equation (4.1c) implies that there exists a stream function $\psi$ which we assume is given as the initial data. Then by defining $\phi=f_{0} \psi$ we see the constraints of (4.1) are satisfied. Unfortunately this approach is not compatible with the related Cauchy problem. For example, (3.31a) and (4.1b) uniquely determine $u$ and $\phi$ at $x=0$. Clearly we want these values to be the same as those of the related Cauchy problem. However unless $b(0 y, 0)=0$ in the related Cauchy problem, this will not be the case. Assuming $c=0$ also puts an unnecessary restriction on $u$, namely that $\int_{0}^{2 \pi}[u]_{0}^{1} \mathrm{~d} y=0$. Clearly these problems arise since $a, b$, and $c$ have been assumed to be zero which in general they need not be.

At this point in the bounded derivative method, we do not know $a, b$, and $c$ (unless the related Cauchy problem has already been solved) and we assume that they are chosen so that (4.1) in conjunction with (3.31) and (3.32) has a solution. Then we know that the system of constraints (4.1) has one degree of freedom. We can choose the free variable in two ways. For the first approach we follow the pattern suggested by the one-dimensional characteristic variables. For the one-dimensional case, $v$ is the variable which is varying on the slow time scale and so we assume $v$ is given initially in such a way that $(3.31 \mathrm{~b})$ is satisfied. Then we can integrate (4.1a) and (4.1c) with respect to $x$ since we know $\phi(0 y, t)$ and $u(0, y, t)$. Equation (4.1b) is also satisfied since $b$ was chosen so that it is satisfied on the boundary and (4.1c) ensures it is satisfied on the interior.

In the second approach, we utilize concepts which are more consistent with fluid dynamical notions. Here we assume the initial vorticity $\zeta$ is given. The value of $v$ at $x=0$ is given by $(3.31 \mathrm{~b}$ ) and we can specify $v(1, y, 0)$ freely as long as there is smoothness of $v(1, y, 0)$ with respect to $y$. Since the divergence $\delta=u_{x}+v_{y}$ is given by (4.1c), we can solve for the velocity components $u$ and $v$ using the Helmholtz relations

$\nabla^{2} u=\delta_{x}-\zeta_{y}$,
$\nabla^{2} v=\delta_{y}+\zeta_{x}$, since we know $u$ and $v$ at $x=0$ and $x=1$. Notice that once $c$ is specified, $v$ can be determined independently of $u$ and $\phi$. The reason for this uncoupling is that the variable $v$ is not coupled to the other two variables through the boundary conditions. Finally we can differentiate (4.1a) and (4.1b) with respect to $x$ and $y$, respectively, and then add to obtain the equation

$\nabla^{2} \phi=f_{0} \zeta+\varepsilon\left(a_{x}+b_{y}\right)$

which can be solved uniquely for $\phi$ since we know $\phi_{x}(0, y, 0)$ and $\phi_{x}(1, y, 0)$.

We know that requiring the first-order time derivatives to be of order unity does not sufficiently control the gravity waves. By requiring the second-order time derivatives to be of order unity we obtain constraints which can be used to determine $a, b$, and $c$ to order unity. For the external mode, we can replace the divergence $\delta$ in (4.4) and (4.5) by $\varepsilon^{2} c$ and not much is added to the determination of $u$ and $v$ by actually computing $c$. Also in the constraint (4.1d) we can drop the term involving $c$ if we only wish to compute $a$ and $b$ to order unity. With these assumptions, it is not necessary to compute $c$ and one constraint we obtain from the second-order time derivative together with (4.1d) form the uncoupled system for $a$ and $b$ (Browning et al., 1980)

$$
\begin{aligned}
& a_{x}+b_{y}=-\beta y \zeta+\beta u^{0}-2\left(u_{x}^{0} v_{y}^{0}-u_{y}^{0} v_{x}^{0}\right), \\
& -a_{y}+b_{x}=0
\end{aligned}
$$

where the superscript 0 means the nondivergent portion of the wind. Equation (4.7b) implies that the vector $(a, b)^{l}$ is the gradient of a scalar function which we call $\tilde{\phi}$. Then $(4.7 \mathrm{~b})$ is automatically satisfied and $(4.7 \mathrm{a})$ is just a Poisson equation for $\bar{\phi}$. Since we have assumed nondivergent initial wind data, we can use for $\tilde{\phi}$ the same boundary conditions as in Section 3, i.e.

$$
\begin{aligned}
& \delta_{x}(0, y, t)=-\left(u_{t}-\bar{u} v_{y}^{0}+\bar{v} u_{y}^{0}\right)(0, y, t), \\
& \oint_{x}(1, y, t)=-\left(u_{1}-\bar{u} v_{y}^{0}+\tilde{v} u_{y}^{0}\right)(1, y, t),
\end{aligned}
$$

and everything on the right-hand side of (4.8) is known on the boundary. We choose the unique solution whose mean is zero. Improved values for $\phi$ can now be obtained by solving the equation

$\nabla^{2} \phi=f_{0} \zeta+\varepsilon \nabla \delta$ 
with the boundary conditions

$$
\begin{aligned}
& \left(\phi_{x}-f_{0} v^{0}\right)(0, y, t)=\varepsilon \tilde{\phi}_{x}(0, y, t), \\
& \left(\phi_{x}-f_{0} v^{0}\right)(1, y, t)=\varepsilon \tilde{\phi}_{x}(1, y, t),
\end{aligned}
$$

and choose the unique solution whose mean is the same as that of the observed data for $\phi$.

\section{Numerical results}

To test the initialization scheme we derived in Section 4, we consider system (3.1) in the region

$R=\{(x, y): 0 \leqslant x \leqslant X,-Y \leqslant y \leqslant Y\}$.

At the boundaries $y=-Y$ and $y=Y$ we assume solid wall boundary conditions, i.e. the normal component of the velocity $v$ is required to be zero for these values of $y$. We consider two types of boundary conditions for the boundaries at $x=0$ and $x=X$. For what we call the global problem, we assume periodic boundary conditions in the $x$ direction. For what we call the limited area problem, we assume open boundary conditions at $x=0$ and $x=X$. In the corresponding numerical models which we will describe next, we actually used the dimensional form of (3.1) and the corresponding equations can be obtained by setting $\varepsilon=1$. We leave the parameter $\varepsilon$ in the equations for consistency with Sections 3 and 4.

To discretize the two initial-boundary value problems, we choose spatial increments $\Delta x=X / I$ and $\Delta y=2 Y / J$, where $I$ and $J$ are natural numbers. The temporal increment $\Delta t$ is determined from the stability requirement. We define the finite difference grid

$$
\begin{aligned}
G & =\left\{\left(x_{i}, y_{j}, t_{n}\right): x_{i}=(i-1) \Delta x, y_{J}\right. \\
& \left.=-Y+(j-1) \Delta y, t_{n}=n \Delta t\right\}
\end{aligned}
$$

with $1 \leqslant i \leqslant I+1,1 \leqslant j \leqslant J+1$, and $-1 \leqslant n \leqslant N$ and employ the standard grid function notation $u_{i, j}^{n}$ $\approx u\left(x_{i}, y_{j}, t_{n}\right)$. We assume that missing subscripts or superscripts are the nominal values $i, j$, or $n$ as appropriate. By defining the difference operators

$$
\begin{aligned}
& \mathrm{D}_{t} u=\frac{u^{n+1}-u^{n-1}}{2 \Delta t}, \\
& \mathrm{D}_{x} u=\frac{u_{i+1}-u_{i-1}}{2 \Delta x}, \\
& \mathrm{D}_{y} u=\frac{u_{j+1}-u_{j-1}}{2 \Delta y},
\end{aligned}
$$

we can approximate system (3.1) at time $t=n \Delta t$ at each grid point in the interior of $R(2 \leqslant i \leqslant I$ and $2 \leqslant j \leqslant J)$ by

$$
\begin{aligned}
& \mathrm{D}_{t} u+u \mathrm{D}_{x} u+v \mathrm{D}_{y} u+\varepsilon^{-1}\left[\mathrm{D}_{x} \phi\right. \\
& \left.-\left(f_{0}+\beta y\right) v\right]=0
\end{aligned}
$$

$$
\begin{aligned}
& \mathrm{D}_{t} v+u \mathrm{D}_{x} v+v \mathrm{D}_{y} v+\varepsilon^{-1}\left[\mathrm{D}_{y} \phi\right. \\
& \left.+\left(f_{0}+\beta y\right) u\right]=0,
\end{aligned}
$$

$\mathrm{D}_{t} \phi+u \mathrm{D}_{x} \phi+v \mathrm{D}_{y} \phi+\varepsilon^{-2}\left(\phi_{0}+\varepsilon^{2} \phi\right)$

$$
\times\left(\mathrm{D}_{x} u+\mathrm{D}_{y} v\right)=0 \text {. }
$$

For $j=1$ and $j=J+1$, the equation for $u$ can be used as before since the term $v \mathrm{D}_{\mathrm{y}} u$ is zero there. The equation for $v$ is not required as $v=0$ on these boundaries. In the equation for $\phi$, the term $v \mathrm{D}_{\mathrm{y}} \phi$ is zero and we approximate the term $v_{y}$ by the appropriate one-sided second-order difference formula. When applying (5.1) for $i=1$ in the global model, variables with a zero subscript are determined from the periodicity conditions e.g. $u_{0, J}=$ $u_{I, j}$. All variables at $i=I+1$ are set to their corresponding value at $i=1$. For the limited area model we used the one-dimensional characteristic variable method investigated by Elvius and Sundström (1973). We will only describe this method at $x=0$ since the approximations used at $x=X$ can be derived in an analogous fashion. For this method we define the operator

$\mathrm{D}_{x} u=\frac{2 u_{i+1}-\left(u^{n+1}+u^{n-1}\right)}{2 \Delta x}$.

We introduce this operator because if we approximate the half plane problem

$u_{t}=u_{x} \quad 0 \leqslant x<\infty$,

by $\mathrm{D}_{t} u=\mathrm{D}_{x} u$ on the interior points and by $\mathrm{D}_{t} u=$ $\mathrm{D}_{x} u$ at $x=0$, then the approximation is known to be stable. If we have inflow at $x=0$, i.e. $u(0, y t)>$ 0 , then we assume that the value of $v(0, y, t)$ is given. If we have outflow at $x=0$, i.e. $u(0, y, t) \leqslant 0$, then we compute $v$ by $(5.1 \mathrm{~b})$ with the operator $\mathrm{D}_{x}$ replaced by $\tilde{\mathrm{D}}_{x}$. We also assume that the linear combination of $u$ and $\phi$ in (3.31a) is given at $x=0$, i.e.

$u(0, y, t)=-\varepsilon^{2} \phi(0, y, t)+g_{0}(y, t)$.

Then $u$ and $\phi$ are computed at $i=1$ from (5.1a) and $(5.1 \mathrm{c})$ with the operator $\mathrm{D}_{x}$ replaced by $\mathrm{D}_{x}$. 
This substitution results in $a 2 \times 2$ coupled system for $u_{1, j}^{n+1}$ and $\phi_{1, j}^{n+1}$. The system is uncoupled by solving (5.2) for $\phi(u)$ at $t=(n+1) \Delta t$ and substituting the result for $\phi_{1, j}^{n+1}\left(u_{1, j}^{n+1}\right)$ in (5.1a) $((5.1 \mathrm{c}))$. The values of those variables needed on the boundaries are obtained by a prior run of the global model with the same initial conditions.

To determine balanced initial conditions for system (5.1), we assume that the given initial data are the nondivergent wind components $u^{0}$ and $v^{0}$ and the corresponding vorticity $\zeta$. For the external mode, we do not need to calculate the divergent component of the wind to obtain smooth solutions. We compute the initial geopotential by combining equations (4.6) and (4.7a) to obtain the elliptic equation for $\phi$

$\nabla^{2} \phi=f_{0} \zeta+\varepsilon\left[\beta y \zeta-\beta u^{0}+2\left(u_{x}^{0} v_{y}^{0}-u_{y}^{0} v_{x}^{0}\right)\right]$

The boundary conditions at $y=-Y$ and $y=Y$ are derived similar to $(4.8 \mathrm{a})$, i.e. we differentiate the boundary condition $v=0$ and then use the result in the second equation of (3.3) so that on these boundaries

$\phi_{y}=-f_{0} u^{0}-\varepsilon \beta y u^{0}$.

For the global model we used periodic boundary conditions in the $x$ direction. For the limited area model we assume that the geopotential deviation $\phi$ on the boundaries satisfies (4.8a) and (4.9b), i.e.

$$
\begin{aligned}
& \phi_{x}(0, y, 0)=\left[f_{0} v^{0}-\varepsilon\left(u_{t}+u^{0} u_{x}^{0}+v^{0} u_{y}^{0}-\beta y v^{0}\right)\right] \\
& \quad \times(0, y, 0), \\
& \phi_{x}(1, y, 0)=\left[f_{0} v^{0}-\varepsilon\left(u_{t}+u^{0} u_{x}^{0}+v^{0} u_{y}^{0}-\beta y v^{0}\right)\right] \\
& \quad \times(1, y, 0),
\end{aligned}
$$

where the $u_{t}$ terms in $(5.3 \mathrm{c})$ and $(5.3 \mathrm{~d})$ can be replaced by $(\partial / \partial t) g_{0}$ and $(\partial / \partial t) g_{1}$, respectively. In both the global and limited area models equation (5.3a) with the appropriate boundary conditions uniquely determines $\phi$ up to a constant. For the global model this constant is chosen so that the mean of $\phi$ is zero. In the limited area case the mean would be set to the mean of the observed values. In our case the mean was set to zero since the global and limited area model have the same domain. In both models the elliptic equation is solved by the direct methods of Swarztrauber and Sweet (1975). Their package requires the right-hand side of (5.3a) for the grid points which are in the interior of $R$.
We computed those values by replacing the operators $\partial / \partial x$ and $\partial / \partial y$ by $\mathrm{D}_{x}$ and $\mathrm{D}_{y}$, respectively. Since $(5.3 \mathrm{~b})$ is a Neumann boundary condition, their package also requires the righthand side of (5.3a) at $y=-Y$ and $y=Y$. We replaced the $y$ derivatives on those boundaries by second-order one-sided difference formulas. A similar technique was used for $x=0$ and $x=1$ for the limited area boundary conditions $(5.3 \mathrm{c})$ and (5.3d).

For the numerical results which follow, we obtained the initial nondivergent wild components from the stream function $\psi$ given by

$\psi--u_{0} y-u_{1} Y \sin k_{1} x\left[1-\cos k_{2}(y+Y)\right] / \pi$,

where $u_{0}$ and $u_{1}$ are constants, $k_{1}=2 \pi / X$, and $k_{2}=$ $\pi / Y$. The corresponding nondivergent wind components are

$u^{0}=-\psi_{y}=u_{0}+u_{1} \sin k_{1} x \sin k_{2}(y+\eta)$.

$v^{0}=\psi_{x}=-2 u_{1} Y \cos k_{1} x\left(1-\cos k_{2}(y+Y) \mid / X\right.$.

The parameters of the numerical model have the values:

$$
\begin{array}{rlrl}
X & =4000 \mathrm{~m}, & Y & =2000 \mathrm{~km}, \\
I & =40, & J & =40, \\
u_{0} & =20 \mathrm{~m} \mathrm{~s}^{-1}, & u_{1} & =10 \mathrm{~m} \mathrm{~s}^{-1}, \\
\phi_{0} & =10^{5} \mathrm{~m}^{2} \mathrm{~s}^{-2}, & & \\
f_{0} & =2 \Omega \sin (\pi / 4) \mathrm{s}^{-1}, & \beta & =2 \Omega \cos (\pi / 4) / r(\mathrm{~m} \mathrm{~s})^{-1}, \\
\Omega & =7.292 \times 10^{-5} \mathrm{~s}^{-1}, r & =6.37122 \times 10^{6} \mathrm{~m} .
\end{array}
$$

Fig. 1 is a contour map of the initial vorticity derived from (5.4) with the contour values given in units of $10^{-5} \mathrm{~s}^{-1}$.

To check the theory of Sections 3 and 4, we first ran the global model with the initial velocity components taken from (5.5). The initial geopotential deviation was determined from (5.3a) with $\phi$ satisfying periodic boundary conditions in $x$ and (5.3b) on the $y$ boundaries. Using the values of $u, v$, and $\phi$ from this run we determined values for $g_{0}, g_{1}$, and $h$ from (3.31) and (3.32). In the first limited area experiment we used the nondivergent velocity components (5.5) as initial data for $u$ and $v$. The initial values of $\phi$ were derived from (5.3a) with boundary conditions ( $5.3 \mathrm{~b} \mathrm{c}, \mathrm{d}$ ) and were virtually indistinguishable from the initial values of $\phi$ for the global model (see Section 3). The boundary 


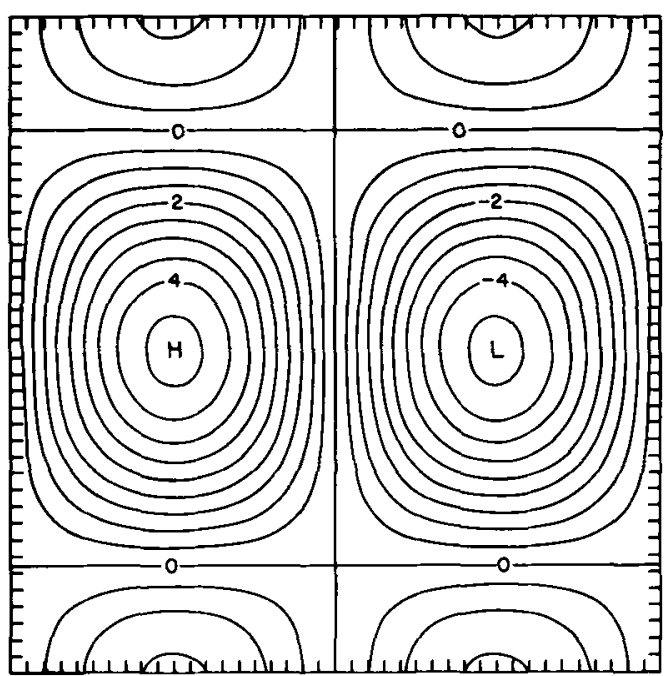

Fig. 1. Initial vorticity for both global and limited area models derived from (5.4). Contour values are given in units of $10^{-9} s^{-1}$.

conditions for the model were those given by (3.31) and (3.32) with the $g_{0} g_{1}$, and $h$ determined above. Fig. 2 is a plot of the geopotential deviation $\phi$ from this run of the limited area model in units of $10^{2} \mathrm{~m}^{2}$ $\mathrm{s}^{-2}$ versus time $t$ in hours for the two grid points $A$ with $(i, j)=(11,11)$ and B with $(i, j)=(21,21)$. The magnitude of the gravity wave oscillations is no larger than that of the global model, i.e. the open boundary conditions (3.31)-(3.32) are not a source of excitation of gravity waves. Fig. 3 is a contour map of the vorticity at $t=48$ hours with the contour values given in units of $10^{-5} \mathrm{~s}^{-1}$ for the global model. We do not show the corresponding map for the limited area model since the two maps are identical to the resolution of a contour map. The relative $L^{2}$ error had the value

$$
\frac{\left\|\zeta_{\text {global }}-\zeta_{\text {limited }}\right\|}{\left\|\zeta_{\text {global }}\right\|}=3.3 \times 10^{-3}
$$

In the second limited area experiment we used the same initial values but maintained $g_{0} g_{1}$, and $h$ at their initial values. Fig. 4 is a plot of the geopotential deviation $\phi$ from this run of the limited area model in units of $10^{2} \mathrm{~m}^{2} \mathrm{~s}^{-2}$ versus time $t$ in hours for the two grid points A with $(i, j)=(11,11)$ and $\mathrm{B}$ with $(i, j)=(21,21)$. Notice that the magnitude of the gravity wave oscillations is no larger than in the first experiment. Figs. 5a and 6a are maps of the geopotential deviation $\phi_{\text {global }}$ and the vorticity $\zeta_{\text {global }}$

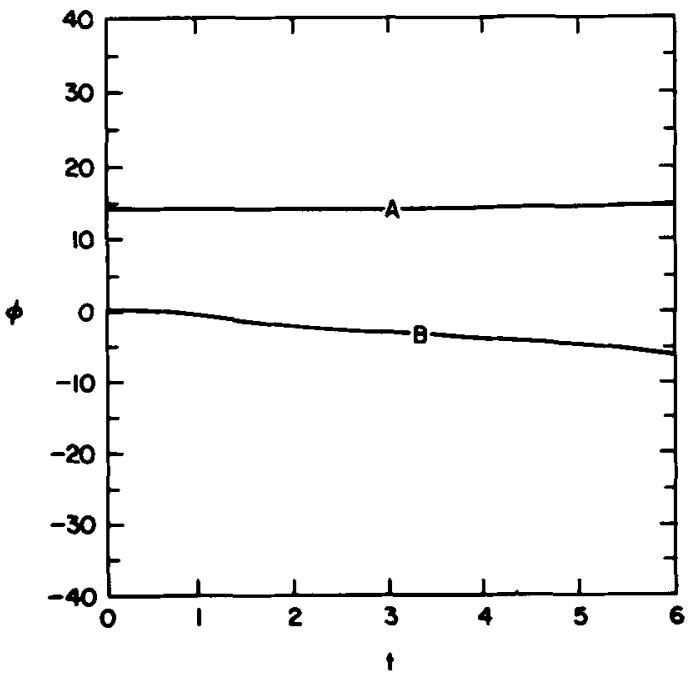

Fig. 2. Geopotential deviation $\phi$ in units of $10^{2} \mathrm{~m}^{2} \mathrm{~s}^{-2}$ given as a function of time in hours for the first limited area experiment. Curve $\mathbf{A}$ and curve $\mathbf{B}$ are the deviation at grid points $(i, j)=(11,11)$ and $(i, j)=(21,21)$ respectively.

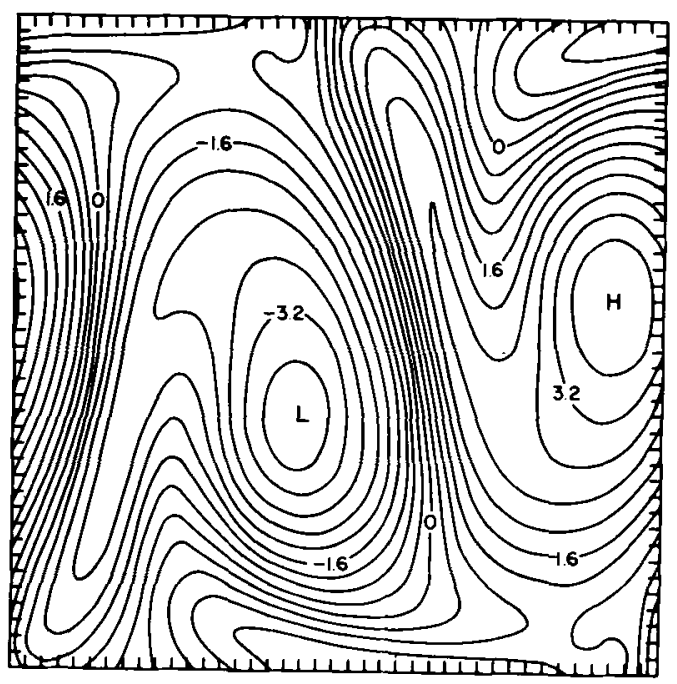

Fig. 3. Vorticity $\zeta$ of global model plotted at $t=48$ hours. Contour values are given in units of $10^{-5} \mathrm{~s}^{-1}$.

from the global model at $t=6$ hours. The contour values are given in units of $10^{3} \mathrm{~m}^{2} \mathrm{~s}^{-2}$ and $10^{-5} \mathrm{~s}^{-1}$, respectively. Finally Figs. $5 \mathrm{~b}$ and $6 \mathrm{~b}$ are the error maps $\phi_{\text {global }}-\phi_{\text {limited }}$ and $\zeta_{\text {global }}-\zeta_{\text {limited }}$ for the second limited area experiment at $t=6$ hours. The contour values for $5 b$ are 0.1 of those for $5 a$ while those of $6 \mathrm{~b}$ are the same as those of $6 \mathrm{a}$. Even 


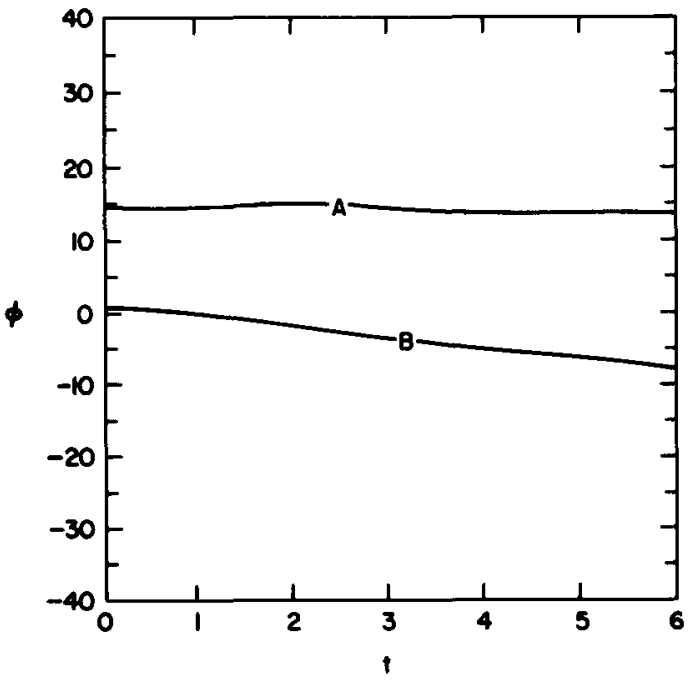

Fig. 4. Geopotential deviation $\phi$ in units of $10^{2} \mathrm{~m}^{2} \mathrm{~s}^{-2}$ given as a function of time in hours for the second limited area experiment. Curve $\mathbf{A}$ and curve $B$ are the deviation at grid points $(i, j)=(11,11)$ and $(i, j)=(21,21)$, respectively.

though the geopotential is smooth in both space and time, it has a relative error of approximately $10 \%$ spread throughout the grid G. However, as might be expected from the form of the vorticity equation, the large error in the vorticity is confined near the boundary.

(a)

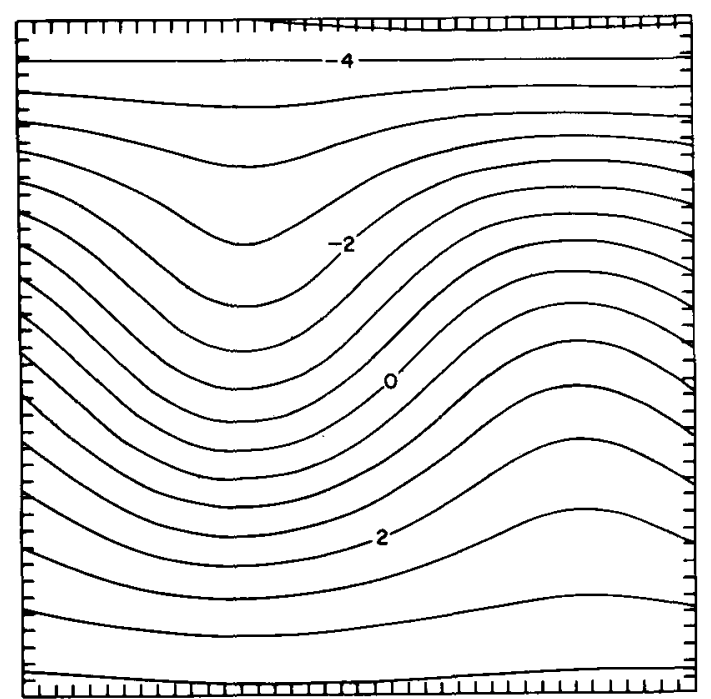

\section{Conclusions}

In this paper we have proven the existence of smooth solutions of the shallow water equations in a region $R$ with open boundary conditions. The importance of the form of the homogeneous boundary conditions and the smoothness of the inhomogeneous terms of the boundary conditions in maintaining the smoothness of the solution is evident in that proof. The inhomogeneous terms are certainly smooth if they are derived from the smooth solution of the related Cauchy problem, i.e. from the smooth solution of the system in a region $\tilde{R}$ which contains $R$ and with periodic boundary conditions. In that case we proved that if the initial conditions for the open boundary problem are chosen as the proper restriction of the initial values of the smooth Cauchy solution then it is possible to reproduce the Cauchy solution in $R$. If the inhomogeneous terms are incorrect but smooth then we also showed that the ensuing solution was smooth. Unfortunately the error propagates at the inertial/gravity wave speed so that the boundary data errors must be kept small. Since for smooth solutions the geopotential basically satisfies the elliptic equation (3.15) it can be shown that only the large-scale boundary data need be correct, i.e. the effect of small-scale errors decays exponen-

(b)

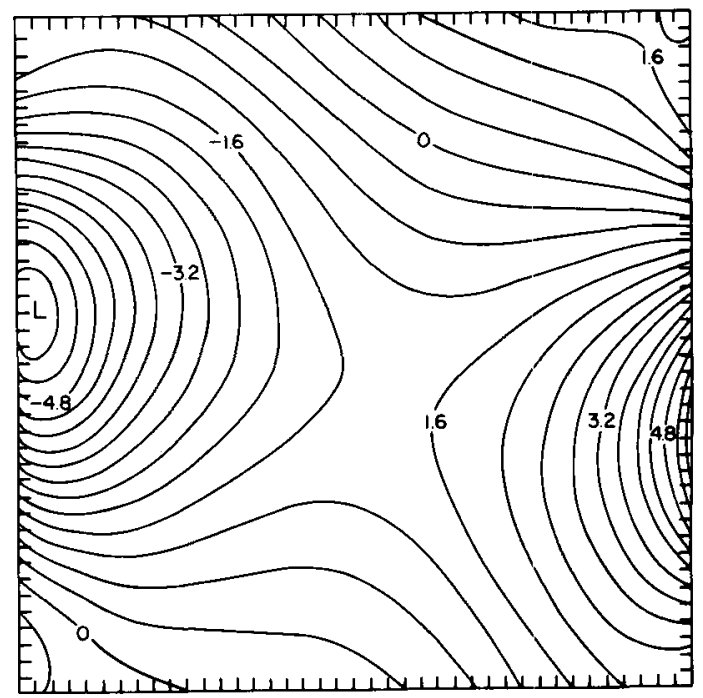

Fig. 5. (a) Geopotential deviation of global model $\phi_{\text {global }}$ and (b) the error in the second Iimited area experiment $\phi_{\text {giobal }}$ $-\phi_{\text {limited }}$ plotted at $t=6$ hours. Contour values are given in units of $10^{3} \mathrm{~m}^{2} \mathrm{~s}^{-2}$ for (a) and units of $10^{2} \mathrm{~m}^{2} \mathrm{~s}^{-2}$ for (b). 
(a)

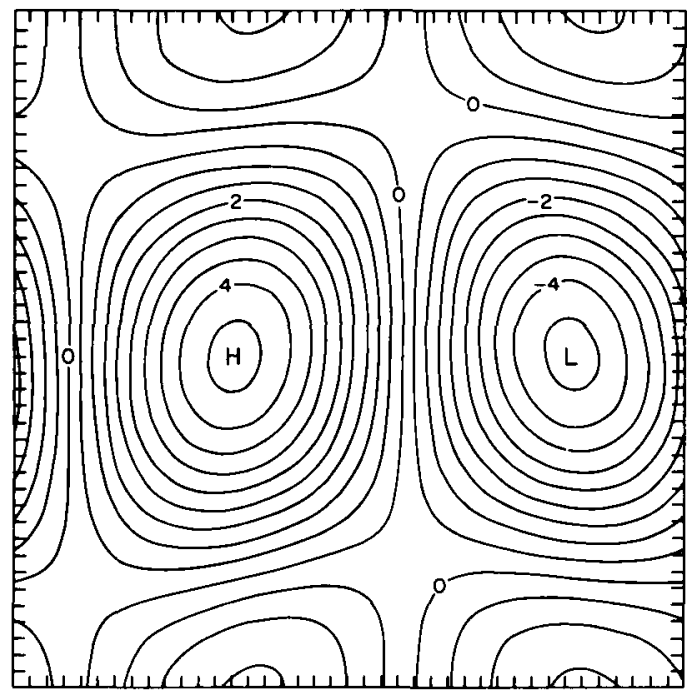

(b)

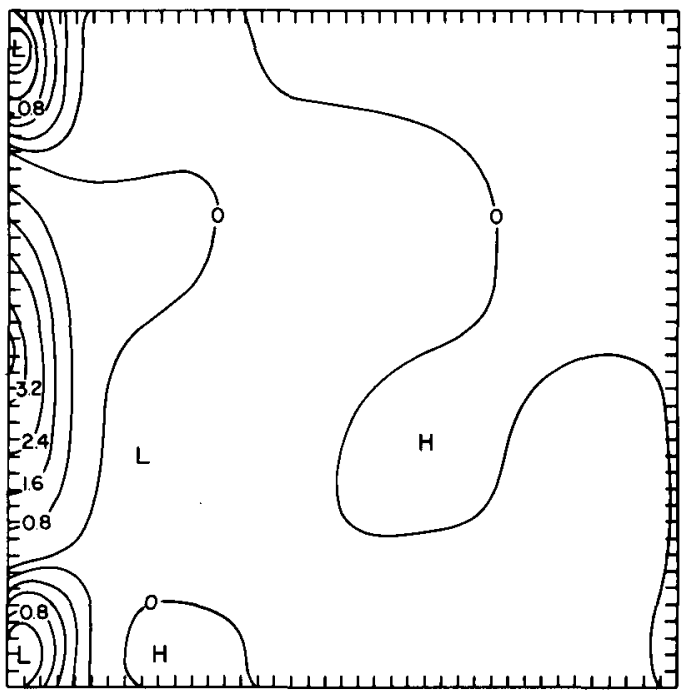

Fig. 6. (a) Vorticity of global model $\zeta_{\text {global }}$ and (b) the error in the second limited area experiment $\zeta_{\text {global }}-\zeta_{\text {1lmited }}$ plotted at $t=6$ hours. Contour values are given in units of $10^{-5} \mathrm{~s}^{-1}$.

tially away from the boundary. The open boundary conditions were an integral part of the energy proof and thus necessarily, of the corresponding initialization procedure. We showed how to incorporate the open boundary conditions into that procedure.

These techniques cannot be applied to the primitive equations as they are not a symmetric hyperbolic system. However, in a forthcoming paper we will apply the bounded derivative method to the Eulerian equations from which the primitive equations are derived. In that paper we derive a system of equations which describes the large-scale solutions of the atmosphere very accurately and are well posed for the open boundary problem (the primitive equations do not have this propertyOliger and Sundström 1978). As one component of the new system essentially behaves like shallow water equations, the results of this paper will be directly applicable to that portion of the new system.

\section{REFERENCES}

Berger, M., Gropp, W. and Oliger J. 1981. Adaptive methods for hyperbolic partial differential equations. Stanford University Numerical Analysis Project Manuscript NA-81-18.

Browning G., Kasahara A. and Kreiss H.-O. 1980. Initialization of the primitive equations by the bounded derivative method. J. Atmos. Sci. 37, 1424-1436.

Elvius T. and Sundström, A. 1973. Computationally efficient schemes and boundary conditions for a fine-mesh model based on the shallow-water equations. Tellus 25, 132-156.

Gustafsson B. and Kreiss, H.-O. 1980. Difference approximations of hyperbolic problems with different time scales I. The reduced problem. Uppsala University Department of Computer Science Report 86.

John F. 1952. Partial differential equations. CIMS Lecture Notes, Courant Institute of Mathematical Sciences, 251 Mercer Street, New York, N.Y. 10012. 248 pp.

Kreiss, H.-O. 1970. Initial boundary value problems for hyperbolic differential equations. Comm. Pure Appl. Math. 23, 277-298.

Kreiss, H.-O. 1980. Problems with different time scales for partial differential equations. Comm. Pure Appl. Math. 33, 399-440.

Oliger, J. and Sundström, A. 1978. Theoretical and practical aspects of some initial-boundary value problems in fluid dynamics. SIAM J. Appl. Math. 35 , $419-446$.

Sundström, A. and Elvius, T. 1979. Computational problems related to limited-area modeling. GARP Publications Series, 17, 379-416.

Swarztrauber, P. and Sweet, R. 1975. Efficient FORTRAN subprograms for the solution of elliptic partial differential equations. NCAR-TN/IA-109. National Center for Atmospheric Research, Boulder. Colo., 139 pp.

Williamson, D. and Dickinson, R. 1976. Free oscillations of the NCAR global circulation model. Mon. Wea. Rev. I04, 1372-1391.

Tellus 34 (1982), 4 